\title{
THE $x$-DIVISOR PSEUDOGRAPHS OF A COMMUTATIVE GROUPOID
}

\author{
John D. LaGrange \\ Received: 25 October 2016; Revised: 22 December 2016 \\ Communicated by Burcu Üngör
}

\begin{abstract}
The notion of a zero-divisor graph is considered for commutative groupoids with zero. Moufang groupoids and certain medial groupoids with zero are shown to have connected zero-divisor graphs of diameters at most four and three, respectively. As $x$ ranges over the elements of a commutative groupoid $\mathcal{A}$ (not necessarily with zero), a system of pseudographs is obtained such that the vertices of a pseudograph are the elements of $\mathcal{A}$ and vertices $a$ and $b$ are adjacent if and only if $a b=x$. These systems are completely characterized as being partitions of complete pseudographs $\bar{K}_{n}$ whose parts are indexed by the vertices of $\bar{K}_{n}$. Furthermore, morphisms are defined in the class of all such systems of pseudographs making it (categorically) isomorphic to the category of commutative groupoids, thereby combinatorializing the theory of commutative groupoids. Also, concepts of "congruence" and "direct product" that are compatible with those in the category of commutative groupoids are established for these systems of pseudographs.
\end{abstract}

Mathematics Subject Classification (2010): 05C25, 18B99, 20N02

Keywords: Groupoid, zero-divisor graph, divisor pseudograph

\section{Introduction}

There are several methods that exist for generating a groupoid (that is, a set endowed with a binary operation ${ }^{1}$ ) from a graph. For example, cycle decompositions of complete graphs are used to produce groupoids in [17], and 1-factorizations of complete graphs are used to construct quasigroups (that is, cancellative groupoids $\mathcal{A}$ such that $a \mathcal{A}=\mathcal{A} a=\mathcal{A}$ for every $a \in \mathcal{A}$, i.e., for every $a, b \in \mathcal{A}$ there exist unique $x, y \in \mathcal{A}$ such that $a x=y a=b)$ in [15] and [22]. The zero-divisor graph of a commutative ring $R$ was first defined in [5] to be the graph $\Gamma(R)$ whose vertices are the elements of $R$ such that distinct $a$ and $b$ are adjacent if and only if $a b=0$. The

\footnotetext{
${ }^{1}$ The present use of the term "groupoid" coincides with the work of B.A. Hausmann and O. Ore in [11] and seems to be standard among a majority of the related work in nonassociative algebra. It should not be confused with the categorical convention in which a groupoid is defined to be a category such that every morphism is invertible.
} 
definition of $\Gamma(R)$ was later revised in [2] to only include the nonzero zero-divisors of $R$, as the algebraic structure reflected by the adjacency relations involving 0 and nonzero-divisors are of little interest. The zero-divisor graph concept was extended to noncommutative rings in [21], commutative semigroups in [9], and partially ordered sets in [10].

In this paper, the notion of a zero-divisor graph is considered for commutative groupoids with 0 . Furthermore, it is extended in a manner that permits any commutative groupoid to be viewed as a partition of a complete pseudograph, where a pseudograph is regarded as a graph without multiple edges that may (but is not required to) have some looped vertices. (Also, to avoid trivialities, the vertex-set $V(G)$ of any pseudograph $G$ will always be assumed nonempty.)

A zero of a commutative groupoid $\mathcal{A}$ is an element $0 \in \mathcal{A}$ such that $a 0=0$ for every $a \in \mathcal{A}$. Clearly a zero of $\mathcal{A}$ is unique. If $\mathcal{A}$ is a commutative groupoid with 0 then define the zero-divisor graph of $\mathcal{A}$ to be the graph $\Gamma(\mathcal{A})$ whose vertices are the elements of $\{a \in \mathcal{A} \backslash\{0\} \mid a b=0$ for some $b \in \mathcal{A} \backslash\{0\}\}$ (i.e., the nonzero zero-divisors of $\mathcal{A}$ ) and distinct vertices $a, b \in \mathcal{A}$ are adjacent if and only if $a b=0$.

Diameter has been a topic of broad interest in the study of zero-divisor graphs (e.g., it has been investigated in zero-divisor graphs of various algebraic structures among [2], [3], [4], [9], [14], [18], [19], and [21], and other contributions have been made that are not reflected in the bibliography of this article). In particular, it was shown in [9] that the zero-divisor graph of a commutative semigroup is connected with diameter at most three. Therefore, the class of graphs that are realizable as zero-divisor graphs of commutative semigroups is relatively "small." In contrast with semigroups, Theorem 2.1 shows that every simple graph is realizable as the zero-divisor graph of a commutative groupoid with 0 . In fact, for every simple graph $G$, the "freest" groupoid with 0 whose zero-divisor graph is $G$ is given.

Nevertheless, it is natural to question the extent to which known results on diameter for zero-divisor graphs of semigroups can be generalized. An obvious approach is to examine more general notions of associativity. For example, a groupoid $\mathcal{A}$ is called a Moufang groupoid if $(a b)(c a)=(a(b c)) a$ for all $a, b, c \in \mathcal{A}$. This condition has been considered extensively for quasigroups (e.g., a Moufang quasigroup was used in a construction of the notorious "monster group" in [7]), and has recently been studied in more general groupoids (see [20]). Theorem 2.2 addresses diameter for commutative Moufang groupoids with 0 . Specifically, it is shown that zero-divisor graphs of commutative Moufang groupoids with 0 are connected with diameter at most four. 
Another well known generalization of associativity is given by the "medial" property. A groupoid $\mathcal{A}$ is called medial (or entropic) if the equality $(a b)(c d)=(a c)(b d)$ holds for every $a, b, c, d \in \mathcal{A}$. Note that medial groupoids with identity are associative (let $b$ be the identity). However, the weaker identity-type condition " $a \in a \mathcal{A}$ for every $a \in \mathcal{A}$ " does not guarantee associativity in a medial groupoid (e.g. let $\mathcal{A}=\mathbb{Q}$ be the set of rational numbers under the operation $a b:=a+a+b+b$, where "+" is the usual addition). Theorem 2.3 shows that if $\mathcal{A}$ is a commutative medial groupoid with 0 such that $a \in a \mathcal{A}$ for every $a \in \mathcal{A}$ then its zero-divisor graph is connected with diameter at most three. We reference [13] for other work on medial groupoids, where the stronger condition " $\mathcal{A}=a \mathcal{A}$ for every $a \in \mathcal{A}$ " is considered.

Let $x$ be an element of a commutative groupoid $\mathcal{A}$. Attempting to extend the definition of a zero-divisor graph in order to arrive at a notion of an " $x$-divisor graph," one might choose the vertex-set to be $\{a \in \mathcal{A} \backslash\{x\} \mid a b=x$ for some $b \in \mathcal{A} \backslash\{x\}\}$. For general $x$, however, algebraic relations involving $x$ may not be clear, i.e., the solutions $a \in \mathcal{A}$ to the equation $a x=x$ might be interesting. Thus, it seems constructive to allow every element of $\{a \in \mathcal{A} \mid a b=x$ for some $b \in \mathcal{A}\}$ to be a vertex. Actually, in the present article, we are interested in examining " $x$-divisor graphs" of a given groupoid $\mathcal{A}$ for several different elements $x \in \mathcal{A}$ simultaneously. Comparisons of the relations between different graphs are more meaningful if the graphs have the same vertex-sets, so it will be beneficial to consider every element of $\mathcal{A}$ to be a vertex. Moreover, we will permit looped vertices, and define the $x$-divisor pseudograph of $\mathcal{A}$ to be the pseudograph $\Gamma_{x}(\mathcal{A})$ whose vertices are the elements of $\mathcal{A}$ such that (not necessarily distinct) $a, b \in \mathcal{A}$ are adjacent if and only if $a b=x$.

Let $\mathcal{A}$ be a commutative groupoid. For every $a, b \in \mathcal{A}$, there exists a unique $x \in \mathcal{A}$ such that $a b=x$. Therefore, the complete pseudograph $\bar{K}_{|\mathcal{A}|}$ (i.e., the complete graph on $|\mathcal{A}|$ looped vertices) is given by the union as $x$ ranges over $\mathcal{A}$ of the pseudographs $\Gamma_{x}(\mathcal{A})$. More generally, define a complete system of pseudographs (abbreviated by C.S.P.) to be a $|I|$-tuple $\left\langle G_{i}\right\rangle_{i \in I}$ (where $I$ is an indexing set) of pseudographs $G_{i}$ such that $V\left(G_{i}\right)=I$ for every $i \in I$ and, for all $i, j \in I$, there exists a unique $k \in I$ such that $i$ and $j$ are adjacent in $G_{k}$. Throughout, the C.S.P. $\left\langle\Gamma_{x}(\mathcal{A})\right\rangle_{x \in \mathcal{A}}$ given by a commutative groupoid $\mathcal{A}$ will be denoted by $\mathcal{D}(\mathcal{A})$, and will be referred to as the divisor pseudograph system of $\mathcal{A}$.

Section 3 is devoted to developing a framework for an algebraic theory of C.S.P.s that is compatible with that of commutative groupoids by determining the "correct" definitions of a "C.S.P.-homomorphism" and its "image," a "quotient-C.S.P.," 
and a "direct product" of C.S.P.s. The "homomorphisms" of C.S.P.s defined in Section 3 make the class of C.S.P.s into a category that is isomorphic to the category of commutative groupoids with usual groupoid-homomorphisms (Theorem 3.4(2)). In particular, two commutative groupoids $\mathcal{A}$ and $\mathcal{B}$ are isomorphic if and only if the C.S.P.s $\mathcal{D}(\mathcal{A})$ and $\mathcal{D}(\mathcal{B})$ are "isomorphic" (Theorem 3.4(1)). A notion of "congruence" is given such that an equivalence relation $\sim$ on a commutative groupoid $\mathcal{A}$ is a congruence relation on $\mathcal{A}$ if and only if it induces a "congruence relation" on $\mathcal{D}(\mathcal{A})$, and in this case the C.S.P.s $\mathcal{D}(\mathcal{A} / \sim)$ and $\mathcal{D}(\mathcal{A}) / \sim$ are "isomorphic" (in fact, they are equal; Theorem 3.7). Moreover, a "direct product" of C.S.P.s is defined such that, for a family $\left\{\mathcal{A}_{j}\right\}_{j \in J}$ of commutative groupoids, the C.S.P.s $\mathcal{D}\left(\prod_{j \in J} \mathcal{A}_{j}\right)$ and $\prod_{j \in J} \mathcal{D}\left(\mathcal{A}_{j}\right)$ are "isomorphic" (in fact, they are equal), where $\prod_{j \in J} \mathcal{A}_{j}$ is the usual direct product of groupoids (Theorem 3.9).

Throughout, if groupoids $\mathcal{A}$ and $\mathcal{B}$ are isomorphic then we will write $\mathcal{A} \cong \mathcal{B}$. If $x$ is a vertex of a pseudograph $G$ then define $N_{G}(x)=\{y \in V(G) \mid x$ and $y$ are adjacent in $G$ \}. Let $G$ and $G^{\prime}$ be pseudographs. A homomorphism from $G$ into $G^{\prime}$ is a function $\varphi: V(G) \rightarrow V\left(G^{\prime}\right)$ such that $\varphi(a) \in N_{G^{\prime}}(\varphi(b))$ whenever $a \in N_{G}(b)$. The pseudographs $G$ and $G^{\prime}$ are isomorphic, written $G \cong G^{\prime}$ (there will be no risk of confusion with the notation for isomorphic groupoids), if there exists a bijective homomorphism $\varphi: V(G) \rightarrow V\left(G^{\prime}\right)$ such that $\varphi^{-1}: V\left(G^{\prime}\right) \rightarrow V(G)$ is also a homomorphism. A reference on graph theory can be found in [16], and [12] sufficiently addresses the relevant concepts from category theory. Surveys on zero-divisor graphs are given in [1] and [8], and a standard reference on groupoids is $[6]$.

\section{Zero-divisor graphs of commutative groupoids}

While the more general $x$-divisor graph (an idea conceived from the notion of a zero-divisor graph) commands the primary focus of the present article, discussions on this subject seem premature in the absence of any literature on zero-divisor graphs of groupoids. The purpose of this section is to submit a brief introduction to these zero-divisor graphs by considering their diameters in contrast to those of the associative algebraic structures that have already been widely studied. We show that every simple graph is realizable as the zero-divisor graph of a commutative groupoid, and then close the section by presenting examples of results that show the "connected zero-divisor graph" phenomenon extends beyond associative algebra.

Given any nonempty set $X$, let $\mathcal{F}(X)$ be the free commutative groupoid on $X$, i.e., $\mathcal{F}(X)$ is the unique (up to isomorphism) commutative groupoid containing $X$ 
satisfying the following universal property: If $f: X \rightarrow \mathcal{A}$ is any function from $X$ into a commutative groupoid $\mathcal{A}$ then there exists a unique groupoid-homomorphism $\psi: \mathcal{F}(X) \rightarrow \mathcal{A}$ that agrees with $f$ on the set $X$. Note that $\mathcal{F}(X)$ can be regarded as the collection of all finite bracketed products of elements from $X$ such that $a b=x y$ for some $a, b, x, y \in \mathcal{F}(X)$ if and only if $\{a, b\}=\{x, y\}$. Let 0 be an element (not in $\mathcal{F}(X)$ ) and define $\mathcal{F}_{0}(X)=\mathcal{F}(X) \cup\{0\}$ to be the commutative groupoid with zero given by extending the operation on $\mathcal{F}(X)$ so that $0 a=a 0=0$ for every $a \in \mathcal{F}_{0}(X)$.

The following theorem shows that, for every simple graph $G$, there exists a "freest" commutative groupoid $\mathcal{A}$ with 0 whose zero-divisor graph is isomorphic to $G$.

Theorem 2.1. Let $G$ be a simple graph. There exists a commutative groupoid $\mathcal{A}$ with 0 such that $\Gamma(\mathcal{A})=G$, and $\mathcal{A}$ has the following universal property: Let $\iota_{\mathcal{A}}: V(G) \rightarrow \mathcal{A}$ be the inclusion map. If $\mathcal{B}$ is a commutative groupoid with 0 and $\varphi: V(G) \rightarrow V(\Gamma(\mathcal{B})) \subseteq \mathcal{B}$ is a graph-homomorphism then there exists a unique groupoid-homomorphism $\Phi: \mathcal{A} \rightarrow \mathcal{B}$ that agrees with $\varphi$ on $V(G)$, i.e., the following diagram is commutative.

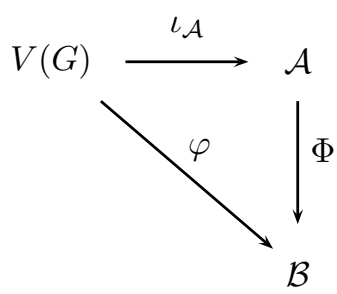

Moreover, $\mathcal{A}$ is unique (up to isomorphism).

Proof. Let $I \subseteq \mathcal{F}_{0}(V(G))$ be the set consisting of 0 , all elements that have a bracketed factor of the form $a b$ for some $a, b \in V(G)$ with $a \in N_{G}(b)$, and all elements that have a bracketed factor of the form $c c$ for some isolated vertex $c \in$ $V(G)$ (i.e., $N_{G}(c)=\emptyset$ ). Define an equivalence relation $\sim$ on $\mathcal{F}_{0}(V(G))$ by $x \sim y$ if and only if either $x=y$ or $x, y \in I$. Set $\mathcal{A}=\left\{\bar{a} \mid a \in \mathcal{F}_{0}(V(G))\right\}$, where $\bar{a}$ denotes the equivalence class of $a$. It is straightforward to check that $\sim$ is a congruence relation on $\mathcal{F}_{0}(V(G))$, and so the operation $\bar{a} \bar{b}=\overline{a b}$ makes $\mathcal{A}$ into a commutative groupoid with zero element $\overline{0}=I$.

By identifying an element $a \in V(G)$ with $\bar{a}$ in $\mathcal{A}$, it is clear that $\Gamma(\mathcal{A})=G$. Let $\mathcal{B}$ be a commutative groupoid with 0 and $\varphi: V(G) \rightarrow V(\Gamma(\mathcal{B})) \subseteq \mathcal{B}$ a graphhomomorphism. Set $\Phi(\overline{0})=0$, and for every finite bracketed product $x$ of elements 
from $V(G)$ let $\Phi(\bar{x})$ be the finite bracketed product in $\mathcal{B}$ obtained from $x$ by replacing every factor $a \in V(G)$ of $x$ with $\varphi(a)$. The resulting function $\Phi: \mathcal{A} \rightarrow \mathcal{B}$ is a well defined (since $\varphi$ is a graph-homomorphism) groupoid-homomorphism such that $\Phi \iota_{\mathcal{A}}=\varphi$, and the verification of the remaining assertions in the theorem are routine: $\Phi$ is unique because $\mathcal{A}$ is generated by (the image of) $V(G)$, and then the uniqueness of $\mathcal{A}$ follows since, for any other $\mathcal{A}^{\prime}$ with $\Gamma\left(\mathcal{A}^{\prime}\right)=G$ that satisfies the universal property, there exist homomorphisms $\Phi_{1}: \mathcal{A} \rightarrow \mathcal{A}^{\prime}$ (by setting $\mathcal{B}=\mathcal{A}^{\prime}$ and $\varphi=\iota_{\mathcal{A}^{\prime}}$ and applying the universal property to $\mathcal{A}$ ) and $\Phi_{2}: \mathcal{A}^{\prime} \rightarrow \mathcal{A}$ (by setting $\mathcal{B}=\mathcal{A}$ and $\varphi=\iota_{\mathcal{A}}$ and applying the universal property to $\mathcal{A}^{\prime}$ ) such that $\Phi_{1} \Phi_{2}$ is the identity homomorphism on $\mathcal{A}^{\prime}$ (by setting $\mathcal{B}=\mathcal{A}^{\prime}$ and $\varphi=\iota_{\mathcal{A}^{\prime}}$ and using the "unique groupoid-homomorphism" portion of the universal property applied to $\mathcal{A}^{\prime}$ ) and $\Phi_{2} \Phi_{1}$ is the identity homomorphism on $\mathcal{A}$ (by setting $\mathcal{B}=\mathcal{A}$ and $\varphi=\iota_{\mathcal{A}}$ and using the "unique groupoid-homomorphism" portion of the universal property applied to $\mathcal{A})$.

The next two theorems show that the well known fact that zero-divisor graphs of commutative semigroups are connected extends to commutative Moufang groupoids with 0 , and commutative medial groupoids with 0 such that $a \in a \mathcal{A}$. Throughout, the distance between two vertices $a$ and $b$ will be denoted by $d(a, b)$, and if $a_{1}, \ldots, a_{n} \in \mathcal{A} \backslash\{0\}$ ( $n$ is a positive integer) then the sequence $\left(a_{1}, \ldots, a_{n}\right)$ will be called a pseudowalk if $a_{i} a_{i+1}=0$ for every $i \in\{1, \ldots, n-1\}$. Note that if $\left(a_{1}, \ldots, a_{n}\right)$ is a pseudowalk then $d\left(a_{1}, a_{n}\right) \leq n-1$.

Theorem 2.2. Let $\mathcal{A}$ be a commutative Moufang groupoid with 0 . Then $\Gamma(\mathcal{A})$ is connected with diameter at most four.

Proof. If $a, b \in V(\Gamma(\mathcal{A}))$ then there exist $x, y \in \mathcal{A} \backslash\{0\}$ such that $a x=0=b y$. The following equalities are derived by using the Moufang property.

(1) $(y x)(a y)=(y[x a]) y=0$.

(2) $(b[a y]) b=(b a)(y b)=0$.

(3) $((x y)[(a y) b])(x y)=((x y)(a y))(b(x y))=((y[x a]) y)(b(x y))=0$.

(4) $((x y)((a y) b))(b(x y))=((x y)[([a y] b) b])(x y)=((x y)[(b a)(y b)])(x y)=0$.

(5) $(b[x y]) b=(b x)(y b)=0$.

(6) $(a[x y]) a=(a x)(y a)=0$.

(7) If $\alpha=a[x y]$ then $(\alpha[a y]) \alpha=(\alpha a)(y \alpha)=((a x)(y a))(y \alpha)=0$.

(8) $((a y)[a(x y)])(a y)=((a y) a)((x y)(a y))=((a y) a)((y[a x]) y)=0$.

(9) $(a y)(b a)=(a[y b]) a=0$.

(10) If $a(a y)=0$ then $(b a)((a y) b)=(b[a(a y)]) b=0$. 
(11) $(a(x y))((a y) a)=(a[(x y)(a y)]) a=(a[(y[x a]) y]) a=0$.

(12) $((a y) b)(a(a y))=((a y)[b a])(a y)=((a[y b]) a)(a y)=0$.

If $a b=0$ then $d(a, b) \leq 1$. Also, if $a y=0$ then $d(a, b) \leq 2$, and if $x y=0$ then $d(a, b) \leq 3$. Therefore, assume that $a b, a y$, and $x y$ are nonzero.

Suppose that $a(x y)=0$. If $b(x y)=0$ then $d(a, b) \leq 2$, so assume $b(x y) \neq 0$. If $(a y) b=0$ then $d(a, b) \leq 3$ since $(a, x y, a y, b)$ is a pseudowalk by (1), so assume $(a y) b \neq 0$. If $(x y)((a y) b)=0$ then $(a, x y,(a y) b, b)$ is a pseudowalk by $(2)$, and if $(x y)((a y) b) \neq 0$ then $(a, x y,(x y)((a y) b), b(x y), b)$ is a pseudowalk by (3), (4), and (5). Thus, $d(a, b) \leq 4$. Henceforth, assume that $a(x y) \neq 0$.

Suppose that $(a y) b=0$. If $(a y)(a(x y))=0$ then $(a, a(x y), a y, b)$ is a pseudowalk by $(6)$, so $d(a, b) \leq 3$. On the other hand, if $(a y)(a(x y)) \neq 0$ then $(a, a(x y),(a y)(a(x y)), a y, b)$ is a pseudowalk by $(6),(7)$, and (8). Hence, $d(a, b) \leq 4$. Therefore, assume that $(a y) b \neq 0$.

Suppose that $a(a y)=0$. Then $(a, a y, a b,(a y) b, b)$ is a pseudowalk by (9), (10), and $(2)$, and hence $d(a, b) \leq 4$. Thus, assume that $a(a y) \neq 0$.

The cases when either $a(x y)=0,(a y) b=0$, or $a(a y)=0$ have been exhausted. But if the elements $a(x y),(a y) b$, and $a(a y)$ are nonzero then $(a, a(x y), a(a y),(a y) b, b)$ is a pseudowalk by (6), (11), (12), and (2). Therefore, $d(a, b) \leq 4$, and the proof is complete.

Relative to the previous result on Moufang groupoids, the case when $\mathcal{A}$ is medial such that $a \in a \mathcal{A}$ for every $a \in \mathcal{A}$ is proved more readily.

Theorem 2.3. Let $\mathcal{A}$ be a commutative medial groupoid with 0 . If a $\in$ a $\mathcal{A}$ for every $a \in \mathcal{A}$ then $\Gamma(\mathcal{A})$ is connected with diameter at most three.

Proof. If $a, b \in V(\Gamma(\mathcal{A}))$ then there exist $x, y \in \mathcal{A} \backslash\{0\}$ such that $a x=0=b y$. If $a y=0$ or $b x=0$ then it is clear that $d(a, b) \leq 2$. Suppose that $a y, b x \neq 0$. Choose $s, t \in \mathcal{A}$ such that $a s=a$ and $b t=b$. Then $a(b x)=(a s)(b x)=(a x)(b s)=0$ and $(b x)(a y)=(b y)(a x)=0$ and $b(a y)=(b t)(a y)=(b y)(a t)=0$, so it follows that $d(a, b) \leq 3$

The following example shows that the assumption " $a \in a \mathcal{A}$ for every $a \in \mathcal{A}$ " cannot be omitted in Theorem 2.3.

Example 2.4. Let ". be the usual multiplication on the integers and set $\mathcal{A}=$ $\{0,1,2,3,4\}$. For all $a, b \in \mathcal{A}$, define $a b=0$ if either $a \cdot b \in\{0,1\}$ or $a \cdot b>5, a b=3$ if $a \cdot b=2$, and $a b=4$ if $a \cdot b \in\{3,4\}$. It is clear that $(x y)(z w)=0=(x z)(y w)$ for all $x, y, z, w \in \mathcal{A}$ (e.g., since $\mathcal{A}^{2}=\{0,3,4\}$, it follows that $\left.\mathcal{A}^{2} \mathcal{A}^{2}=\{0\}\right)$. 
In particular, $\mathcal{A}$ is a commutative medial groupoid with zero element 0 . However, $\Gamma(\mathcal{A})$ is not connected (it is given by the complete graph on $\{2,3,4\}$ together with the isolated vertex 1$)$.

It is clear that the bound given in Theorem 2.3 is sharp (e.g., let $\mathcal{A}$ be the collection of all subsets of $\{1,2,3\}$ under the operation $\cap$ ). However, the following question remains unanswered.

Question 2.5. Does there exist a commutative Moufang groupoid $\mathcal{A}$ with 0 such that $\Gamma(\mathcal{A})$ has diameter four?

\section{Complete systems of pseudographs}

Let $\mathscr{G}_{1}=\left\langle G_{i}\right\rangle_{i \in I}$ and $\mathscr{G}_{2}=\left\langle H_{j}\right\rangle_{j \in J}$ be C.S.P.s. A homomorphism from $\mathscr{G}_{1}$ to $\mathscr{G}_{2}$ is given by a function $\sigma: I \rightarrow J$ such that $\sigma: V\left(G_{i}\right) \rightarrow V\left(H_{\sigma(i)}\right)$ is a homomorphism of pseudographs for every $i \in I$; in this case, the homomorphism of C.S.P.s will be denoted by $\sigma: \mathscr{G}_{1} \rightarrow \mathscr{G}_{2}$. The image $\sigma\left(\mathscr{G}_{1}\right)$ of a homomorphism $\sigma: \mathscr{G}_{1} \rightarrow \mathscr{G}_{2}$ is defined as the $|\sigma(I)|$-tuple $\left\langle\left. H_{j}\right|_{\sigma}\right\rangle_{j \in \sigma(I)}$, where $\left.H_{j}\right|_{\sigma}$ denotes the subpseudograph of $H_{j}$ induced by $\sigma(I)$.

Example 3.1. Let $\mathscr{G}_{1}=\left\langle G_{a}, G_{b}, G_{c}, G_{d}\right\rangle$, where $G_{a}, G_{b}, G_{c}$ and $G_{d}$ are the pseudographs given in Figure 1(a)-(d). Let $\mathscr{G}_{2}=\left\langle H_{x}, H_{y}, H_{z}, H_{w}\right\rangle$, where $H_{x}, H_{y}, H_{z}$, and $H_{w}$ are the pseudographs given in Figure 1(e)-(h). Define $\sigma:\{a, b, c, d\} \rightarrow$ $\{x, y, z, w\}$ by $\sigma(a)=\sigma(b)=y, \sigma(c)=z$, and $\sigma(d)=w$. Then $\sigma$ is a homomorphism from $\mathscr{G}_{1}$ to $\mathscr{G}_{2}$. The homomorphic image of $\sigma$ is $\sigma\left(\mathscr{G}_{1}\right)=\left\langle\left. H_{y}\right|_{\sigma},\left.H_{z}\right|_{\sigma}\right.$, $\left.\left.H_{w}\right|_{\sigma}\right\rangle$, where $\left.H_{y}\right|_{\sigma},\left.H_{z}\right|_{\sigma}$, and $\left.H_{w}\right|_{\sigma}$ are the pseudographs given in Figure 1(i)-(k).

Let $\mathscr{G}_{1}=\left\langle G_{i}\right\rangle_{i \in I}, \mathscr{G}_{2}$, and $\mathscr{G}_{3}=\left\langle H_{j}\right\rangle_{j \in J}$ be C.S.P.s, and suppose that $\sigma$ : $\mathscr{G}_{1} \rightarrow \mathscr{G}_{2}$ and $\tau: \mathscr{G}_{2} \rightarrow \mathscr{G}_{3}$ are homomorphisms. Then $\tau \sigma: V\left(G_{i}\right) \rightarrow V\left(H_{\tau \sigma(i)}\right)$ is a homomorphism of pseudographs for every $i \in I$. Hence, the composition $\tau \sigma: \mathscr{G}_{1} \rightarrow \mathscr{G}_{3}$ of homomorphisms of C.S.P.s is well defined.

Let $\sigma: \mathscr{G}_{1} \rightarrow \mathscr{G}_{2}$ be a homomorphism of C.S.P.s $\mathscr{G}_{1}=\left\langle G_{i}\right\rangle_{i \in I}$ and $\mathscr{G}_{2}=\left\langle H_{j}\right\rangle_{j \in J}$. If $\sigma: I \rightarrow J$ is injective, surjective, or bijective, then $\sigma: \mathscr{G}_{1} \rightarrow \mathscr{G}_{2}$ will be called injective, surjective, or bijective, respectively, and a bijective C.S.P.-homomorphism will be called an isomorphism. The complete systems $\mathscr{G}_{1}$ and $\mathscr{G}_{2}$ are isomorphic, denoted by $\mathscr{G}_{1} \cong \mathscr{G}_{2}$, if there exists an isomorphism $\sigma: \mathscr{G}_{1} \rightarrow \mathscr{G}_{2}$.

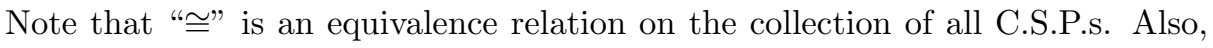
given C.S.P.s $\mathscr{G}_{1}=\left\langle G_{i}\right\rangle_{i \in I}$ and $\mathscr{G}_{2}=\left\langle H_{j}\right\rangle_{j \in J}$, if $\sigma: V\left(G_{i}\right) \rightarrow V\left(H_{\sigma(i)}\right)$ is a bijective homomorphism of pseudographs for every $i \in I$ then so is $\sigma^{-1}: V\left(H_{\sigma(i)}\right) \rightarrow V\left(G_{i}\right)$ (because if $x \in N_{H_{\sigma(i)}}(y)$ but $\sigma^{-1}(x) \notin N_{G_{i}}\left(\sigma^{-1}(y)\right)$ then $\sigma: V\left(G_{k}\right) \rightarrow V\left(H_{\sigma(k)}\right)$ 


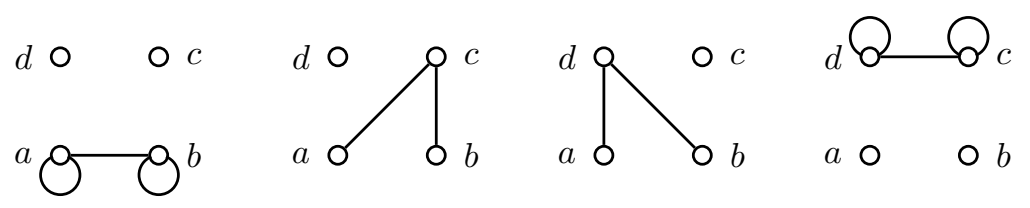
(a) $G_{a}$
(b) $G_{b}$
(c) $G_{c}$
(d) $G_{d}$
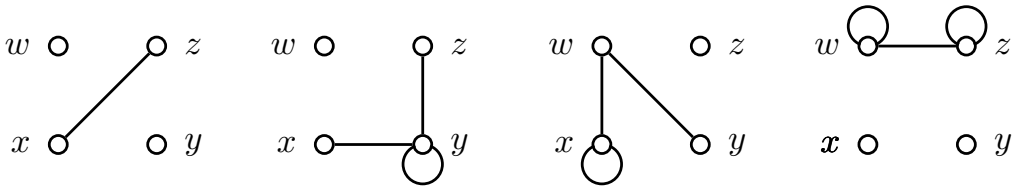

(e) $H_{x}$

(f) $H_{y}$

(g) $H_{z}$

(h) $H_{w}$

$w \circ$

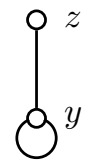

(i) $\left.H_{y}\right|_{\sigma}$

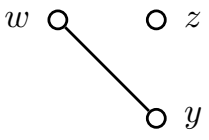

(j) $\left.H_{z}\right|_{\sigma}$

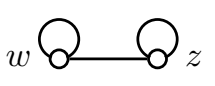

○ $y$

Figure 1. If $\mathscr{G}_{1}=\left\langle G_{a}, G_{b}, G_{c}, G_{d}\right\rangle$ and $\mathscr{G}_{2}=\left\langle H_{x}, H_{y}, H_{z}, H_{w}\right\rangle$ then $\sigma\left(\mathscr{G}_{1}\right)=\left\langle\left. H_{y}\right|_{\sigma},\left.H_{z}\right|_{\sigma},\left.H_{w}\right|_{\sigma}\right\rangle$, where $\sigma: \mathscr{G}_{1} \rightarrow \mathscr{G}_{2}$ is the homomorphism with $\sigma(a)=\sigma(b)=y, \sigma(c)=z$, and $\sigma(d)=w$.

would fail to be a homomorphism, where $k \in I$ is the unique element such that $\sigma^{-1}(x) \in N_{G_{k}}\left(\sigma^{-1}(y)\right)$. This yields the following observation.

Proposition 3.2. Let $\mathscr{G}_{1}=\left\langle G_{i}\right\rangle_{i \in I}$ and $\mathscr{G}_{2}=\left\langle H_{j}\right\rangle_{j \in J}$ be C.S.P.s. If $\sigma: \mathscr{G}_{1} \rightarrow \mathscr{G}_{2}$ is a C.S.P.-isomorphism then $\sigma: V\left(G_{i}\right) \rightarrow V\left(H_{\sigma(i)}\right)$ is a pseudograph-isomorphism for every $i \in I$.

We now begin to establish a dictionary between commutative groupoids and C.S.P.s.

Remark 3.3. (1) Let $\mathcal{A}$ be a commutative groupoid. It has already been observed that $\mathcal{D}(\mathcal{A})=\left\langle\Gamma_{x}(\mathcal{A})\right\rangle_{x \in \mathcal{A}}$ is a C.S.P. Conversely, let $\mathscr{G}=\left\langle G_{i}\right\rangle_{i \in I}$ be a C.S.P. Note that setting $i j=k$ if and only if $i, j, k \in I$ with $i \in N_{G_{k}}(j)$ makes I into a well defined (by the uniqueness of $k$ ) commutative groupoid 
such that $\mathcal{D}(I)=\mathscr{G}$. Moreover, it is clear that if $\mathcal{D}(\mathcal{A})=\mathcal{D}(I)$ for some commutative groupoid $\mathcal{A}$ then $I$ and $\mathcal{A}$ are equal as groupoids.

(2) Suppose that $\sigma: \mathcal{A} \rightarrow \mathcal{B}$ is a homomorphism of commutative groupoids. Let $x \in \mathcal{A}$. If $a, b \in V\left(\Gamma_{x}(\mathcal{A})\right)$ with $a \in N_{\Gamma_{x}(\mathcal{A})}(b)$, i.e., $a b=x$, then $\sigma(a) \sigma(b)=\sigma(a b)=\sigma(x)$, i.e., $\sigma(a) \in N_{\Gamma_{\sigma(x)}(\mathcal{B})}(\sigma(b))$. Hence, for every $x \in \mathcal{A}$, the function $\sigma: V\left(\Gamma_{x}(\mathcal{A})\right) \rightarrow V\left(\Gamma_{\sigma(x)}(\mathcal{B})\right)$ is a homomorphism of pseudographs. Therefore, $\sigma: \mathcal{D}(\mathcal{A}) \rightarrow \mathcal{D}(\mathcal{B})$ is a homomorphism of C.S.P.s.

Conversely, suppose that $\sigma: \mathscr{G}_{1} \rightarrow \mathscr{G}_{2}$ is a homomorphism of C.S.P.s. Then (1) implies that there exist commutative groupoids $\mathcal{A}$ and $\mathcal{B}$ such that $\mathscr{G}_{1}=\mathcal{D}(\mathcal{A})$ and $\mathscr{G}_{2}=\mathcal{D}(\mathcal{B})$. In particular, the homomorphism $\sigma$ is determined by a function $\sigma: \mathcal{A} \rightarrow \mathcal{B}$.

Let $x, y \in \mathcal{A}$. It is evident that $x \in N_{\Gamma_{x y}(\mathcal{A})}(y)$. But $\sigma: V\left(\Gamma_{x y}(\mathcal{A})\right) \rightarrow$ $V\left(\Gamma_{\sigma(x y)}(\mathcal{B})\right)$ is a homomorphism of pseudographs, and therefore $\sigma(x) \in$ $N_{\Gamma_{\sigma(x y)}(\mathcal{B})}(\sigma(y))$. That is, in $\mathcal{B}$, the equality $\sigma(x) \sigma(y)=\sigma(x y)$ holds. Hence, the function $\sigma: \mathcal{A} \rightarrow \mathcal{B}$ is a homomorphism of commutative groupoids.

(3) Let $\mathcal{A}$ and $\mathcal{B}$ be commutative groupoids. It follows from (2) that $\mathcal{A} \cong \mathcal{B}$ if and only if $\mathcal{D}(\mathcal{A}) \cong \mathcal{D}(\mathcal{B})$ (since an isomorphism $\sigma: \mathcal{D}(\mathcal{A}) \rightarrow \mathcal{D}(\mathcal{B})$ of C.S.P.s is, by definition, a homomorphism of C.S.P.s such that $\sigma: \mathcal{A} \rightarrow \mathcal{B}$ is a bijective function).

The discussions in Remark 3.3 are summarized in the next theorem.

Theorem 3.4. The following statements hold.

(1) If $\mathscr{G}$ is a C.S.P. then there exists a unique (up to isomorphism) commutative groupoid $\mathcal{A}$ such that $\mathcal{D}(\mathcal{A}) \cong \mathscr{G}$.

(2) The covariant functor $\mathcal{D}$ from the category of commutative groupoids to the category of C.S.P.s that maps a groupoid $\mathcal{A}$ to its divisor pseudograph system $\mathcal{D}(\mathcal{A})$ and maps a groupoid-homomorphism $\sigma: \mathcal{A} \rightarrow \mathcal{B}$ to the C.S.P.homomorphism $\mathcal{D}(\sigma)=\sigma: \mathcal{D}(\mathcal{A}) \rightarrow \mathcal{D}(\mathcal{B})$ gives an isomorphism of categories.

Proof. Note that (1) is immediate from Remark 3.3(1) and Remark 3.3(3). For (2), it is straightforward to check that the class of C.S.P.s and their homomorphisms satisfy the axioms for a category, and that $\mathcal{D}$ is a covariant functor. From Remark $3.3(2)$, it is easily verified that $\mathcal{D}$ is well defined on morphisms, and that it is full. As $\mathcal{D}(\sigma): \mathcal{D}(\mathcal{A}) \rightarrow \mathcal{D}(\mathcal{B})$ is determined uniquely (by definition) by the function 
$\sigma: \mathcal{A} \rightarrow \mathcal{B}$, it is clear that $\mathcal{D}$ is faithful. But it is observed in Remark 3.3(1) that $\mathcal{D}$ is bijective on objects, and it follows that $\mathcal{D}$ is an isomorphism.

The next result supports the given definition of the "image" of a C.S.P.-homomorphism.

Proposition 3.5. If $\sigma: \mathcal{A} \rightarrow \mathcal{B}$ is a homomorphism of commutative groupoids then $\mathcal{D}(\sigma(\mathcal{A}))=\mathcal{D}(\sigma)(\mathcal{D}(\mathcal{A}))$. In particular, any homomorphic image of a C.S.P. is a C.S.P.

Proof. Note that $\left.\Gamma_{x}(\mathcal{B})\right|_{\sigma}=\Gamma_{x}(\sigma(\mathcal{A}))$ for every $x \in \sigma(\mathcal{A})$. Hence, the equalities $\mathcal{D}(\sigma)(\mathcal{D}(\mathcal{A}))=\left\langle\left.\Gamma_{x}(\mathcal{B})\right|_{\sigma}\right\rangle_{x \in \sigma(\mathcal{A})}=\left\langle\Gamma_{x}(\sigma(\mathcal{A}))\right\rangle_{x \in \sigma(\mathcal{A})}=\mathcal{D}(\sigma(\mathcal{A}))$ hold. It follows that $\mathcal{D}(\sigma)(\mathcal{D}(\mathcal{A})$ ) is the divisor pseudograph system of the groupoid $\sigma(\mathcal{A})$ (cf. Example 3.1). The "in particular" statement holds since $\mathcal{D}$ is surjective on objects and morphisms.

We now turn our attention to determining notions of a "quotient-C.S.P." and a "direct product" of C.S.P.s that are compatible with that of a quotient-groupoid and a direct product of groupoids. Incidentally, as we shall see, such concepts turn out to be closely related to the usual notions of a quotient-pseudograph and a direct product of pseudographs (as defined in [16]). We begin with the concept of quotients.

Let $G$ be a pseudograph. Recall that every equivalence relation $\sim$ on $V(G)$ is regarded as a congruence on $V(G)$, and the corresponding quotient-pseudograph $G / \sim$ is the pseudograph whose vertices are the resulting equivalence classes such that $A \in N_{G / \sim}(B)$ if and only if $a \in N_{G}(b)$ for some $a \in A$ and $b \in B$ (these graph-theoretic congruences and quotients are examined in Chapter 1 of [16]). This definition may seem in contrast to algebraic notions of congruence since relations within $G$ need not be respected by a given equivalence relation (e.g., adjacency between vertices $A$ and $B$ in $G / \sim$ need not reflect adjacency between given representatives $a \in A$ and $b \in B$ in $G$ ). Nevertheless, it will turn out to be suitable for determining the divisor pseudograph system of a quotient-groupoid.

Let $\mathscr{G}=\left\langle G_{i}\right\rangle_{i \in I}$ be a C.S.P. Suppose that $\sim$ is an equivalence relation on $I$, and let $I / \sim$ be the resulting set of equivalence classes. For every $X \in I / \sim$, let $\Gamma_{X}$ be the pseudograph with $V\left(\Gamma_{X}\right)=I / \sim$ and $A \in N_{\Gamma_{X}}(B)$ if and only if $a \in N_{G_{i}}(b)$ for some $a \in A, b \in B$, and $i \in X$ (i.e., $\Gamma_{X}=\cup_{i \in X}\left(G_{i} / \sim\right)$ ). Define the quotient-system $\mathscr{G} / \sim$ of $\mathscr{G}$ to be the $|I / \sim|$-tuple $\left\langle\Gamma_{X}\right\rangle_{X \in I / \sim}$.

Remark 3.6. Observe that $\mathscr{G} / \sim$ need not be a C.S.P. For example, let $\mathscr{G}=\mathcal{D}(\mathcal{A})$ be the C.S.P. determined by the pseudographs given in Figure 2(a)-(c). Let $\sim$ be the 
equivalence relation on $\{a, b, c\}$ whose equivalence classes are given by $\bar{a}=\{a\}$ and $\bar{b}=\bar{c}=\{b, c\}$. Then $\mathscr{G} / \sim$ is not a C.S.P. since $\bar{b} \in N_{\Gamma_{X}}(\bar{b})$ for every $X \in I / \sim$.

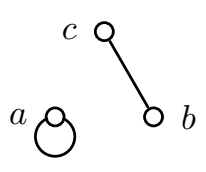

(a) $\Gamma_{a}(\mathcal{A})$

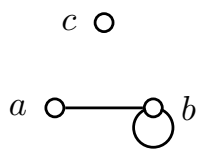

(b) $\Gamma_{b}(\mathcal{A})$

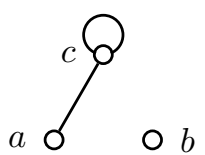

(c) $\Gamma_{c}(\mathcal{A})$

FIGURE 2. If $\sim$ is the equivalence relation on $\{a, b, c\}$ whose equivalence classes are given by $\bar{a}=\{a\}$ and $\bar{b}=\bar{c}=\{b, c\}$ then $\mathscr{G} / \sim$ is not a C.S.P.

Let $\mathscr{G}=\left\langle G_{i}\right\rangle_{i \in I}$ be a C.S.P. An equivalence relation $\sim$ on $I$ will be called a congruence on $\mathscr{G}$ if $\mathscr{G} / \sim$ is a C.S.P. By Theorem 3.4, there exists a functor $\mathcal{D}^{-1}$ from the category of C.S.P.s to the category of commutative groupoids such that $\mathcal{D} \mathcal{D}^{-1}$ and $\mathcal{D}^{-1} \mathcal{D}$ are the identity functors on the categories of C.S.P.s and commutative groupoids, respectively. The next result supports the above definition of a quotient-system.

Theorem 3.7. Given a complete system $\mathscr{G}=\left\langle G_{i}\right\rangle_{i \in I}$ of pseudographs, let $\sim$ be an equivalence relation on $I$. Then $\sim$ is a congruence on $\mathscr{G}$ if and only if $\sim$ is a congruence on the commutative groupoid $I=\mathcal{D}^{-1}(\mathscr{G})$. In this case, $\mathcal{D}(I / \sim)=$ $\mathcal{D}(I) / \sim=\mathscr{G} / \sim$.

Proof. Suppose that $\sim$ is a congruence on $\mathscr{G}$, and let $a, b, x, y \in I=\mathcal{D}^{-1}(\mathscr{G})$ such that $a \sim x$ and $b \sim y$. It is clear that the pseudograph $\Gamma_{\overline{a b}}$ in $\mathscr{G} / \sim$ satisfies $\bar{a} \in N_{\Gamma_{\overline{a b}}}(\bar{b})$. Similarly, $\bar{a}=\bar{x} \in N_{\Gamma_{\overline{x y}}}(\bar{y})=N_{\Gamma_{\overline{x y}}}(\bar{b})$. However, since $\mathscr{G} / \sim$ is a C.S.P., the element $X \in I / \sim$ with $\bar{a} \in N_{\Gamma_{X}}(\bar{b})$ is unique. Hence, $\overline{a b}=X=\overline{x y}$, i.e., $a b \sim x y$. Therefore, $\sim$ is a congruence on the groupoid $I=\mathcal{D}^{-1}(\mathscr{G})$.

To prove the "if" statement, suppose that $\sim$ is a congruence on the commutative groupoid $I=\mathcal{D}^{-1}(\mathscr{G})$. For this portion of the proof, let $\mathcal{D}(I / \sim)=\left\langle\Gamma_{X}(I / \sim)\right\rangle_{X \in I / \sim}$ (as usual), and let $\mathcal{D}(I) / \sim=\left\langle H_{X}\right\rangle_{X \in I / \sim}$, where $H_{X}$ is the pseudograph with $V\left(H_{X}\right)=I / \sim$ and $A \in N_{H_{X}}(B)$ if and only if $a \in N_{G_{i}}(b)$ for some $a \in A, b \in B$, and $i \in X$ (i.e., the notation " $H_{X}$ " is taking the place of " $\Gamma_{X}$ " in the definition of a quotient-system). Since $\mathcal{D}(I / \sim)$ is the divisor pseudograph system of the groupoid $I / \sim$, it is sufficient to prove the second statement of the theorem. 
Note that the equality $\mathcal{D}(I) / \sim=\mathscr{G} / \sim$ holds trivially since $\mathcal{D}(I)=\mathcal{D}\left(\mathcal{D}^{-1}(\mathscr{G})\right)=$ G. To show that $\mathcal{D}(I / \sim)=\mathcal{D}(I) / \sim$, it must be proved that $\Gamma_{X}(I / \sim)=H_{X}$ for every $X \in I / \sim$. Since $V\left(\Gamma_{X}(I / \sim)\right)=I / \sim=V\left(H_{X}\right)$, it suffices to prove that $\Gamma_{X}(I / \sim)$ and $H_{X}$ have the same adjacency relations for every $X \in I / \sim$.

Let $X \in I / \sim$. For every $A, B \in I / \sim$, the containment $A \in N_{\Gamma_{X}(I / \sim)}(B)$ holds if and only if $A B=X$, if and only if $a b \in X$ for every $a \in A$ and $b \in B$. On the other hand, since $\sim$ is a congruence on the groupoid $I$, the containment $a b \in X$ holds for every $a \in A$ and $b \in B$ if and only if $a b=i$ for some $a \in A, b \in B$, and $i \in X$. Hence, $A \in N_{\Gamma_{X}(I / \sim)}(B)$ if and only if $a \in N_{G_{i}}(b)$ for some $a \in A, b \in B$, and $i \in X$; that is, $A \in N_{\Gamma_{X}(I / \sim)}(B)$ if and only if $A \in N_{H_{X}}(B)$. Therefore, $\Gamma_{X}(I / \sim)=H_{X}$.

The following example illustrates a simple application of Theorem 3.7.

Example 3.8. Let $\mathcal{A}=\{a, b, c\}$ be the groupoid given by $a^{2}=b c=a, a b=b^{2}=b$, and $a c=c^{2}=c$. Then $\mathcal{D}(\mathcal{A})$ is given by the pseudographs in Figure $2(a)-(c)$. Define $\sim$ on $\mathcal{A}$ by $\bar{a}=\{a\}$ and $\bar{b}=\bar{c}=\{b, c\}$. By Remark 3.6, $\mathcal{D}(\mathcal{A}) / \sim$ is not a C.S.P. Therefore, by Theorem 3.7, $\sim$ is not a congruence on $\mathcal{A}$.

We close this section by revealing how the functor $\mathcal{D}$ behaves with respect to direct products of groupoids. Let $\left\{G_{j}\right\}_{j \in J}$ be a family of pseudographs. The direct product $\prod_{j \in J} G_{j}$ of $\left\{G_{j}\right\}_{j \in J}$ is the pseudograph whose vertices are the elements of the cartesian product $\prod_{j \in J} V\left(G_{j}\right)$ such that two vertices $\left(a_{j}\right)$ and $\left(b_{j}\right)$ are adjacent if and only if $a_{j}$ and $b_{j}$ are adjacent in $G_{j}$ for every $j \in J$ (what we call a "direct product" is referred to as a "cross product" in [16, Definition 4.2.1]). If $\left\{\mathscr{G}_{j}\right\}_{j \in J}$ is a family of C.S.P.s, say $\mathscr{G}_{j}=\left\langle G(j)_{x}\right\rangle_{x \in I_{j}}(j \in J)$, then define the direct product $\prod_{j \in J} \mathscr{G}_{j}$ of $\left\{\mathscr{G}_{j}\right\}_{j \in J}$ to be the $\left|\prod_{j \in J} I_{j}\right|$-tuple $\left\langle H_{\left(x_{j}\right)}\right\rangle_{\left(x_{j}\right) \in \prod_{j \in J} I_{j}}$, where $H_{\left(x_{j}\right)}=$ $\prod_{j \in J} G(j)_{x_{j}}$. In particular, if $\left\{\mathcal{A}_{j}\right\}_{j \in J}$ is a family of commutative groupoids such that $\mathscr{G}_{j}=\mathcal{D}\left(\mathcal{A}_{j}\right)$ for every $j \in J$ then $H_{\left(x_{j}\right)}=\prod_{j \in J} \Gamma_{x_{j}}\left(\mathcal{A}_{j}\right)$ for all $\left(x_{j}\right) \in$ $\prod_{j \in J} \mathcal{A}_{j}$.

Let $\left(a_{j}\right),\left(b_{j}\right) \in \prod_{j \in J} I_{j}$. For every $j \in J$, there exists a unique $x_{j} \in I_{j}$ such that $a_{j} \in N_{G(j)_{x_{j}}}\left(b_{j}\right)$. It follows that $\left(x_{j}\right)$ is the unique element of $\prod_{j \in J} I_{j}$ such that $\left(a_{j}\right) \in N_{H_{\left(x_{j}\right)}}\left(\left(b_{j}\right)\right)$. Therefore, $\prod_{j \in J} \mathscr{G}_{j}$ is a C.S.P. The next result shows that the functor $\mathcal{D}$ preserves direct products of C.S.P.s.

Theorem 3.9. If $\left\{\mathcal{A}_{j}\right\}_{j \in J}$ is a family of commutative groupoids then the equality $\mathcal{D}\left(\prod_{j \in J} \mathcal{A}_{j}\right)=\prod_{j \in J} \mathcal{D}\left(\mathcal{A}_{j}\right)$ holds. 
Proof. By definition, the C.S.P.s $\mathcal{D}\left(\prod_{j \in J} \mathcal{A}_{j}\right)$ and $\prod_{j \in J} \mathcal{D}\left(\mathcal{A}_{j}\right)$ are both indexed by $\prod_{j \in J} \mathcal{A}_{j}$. Hence, it suffices to verify that

$$
\Gamma_{\left(x_{j}\right)}\left(\prod_{j \in J} \mathcal{A}_{j}\right)=\prod_{j \in J} \Gamma_{x_{j}}\left(\mathcal{A}_{j}\right)
$$

for every $\left(x_{j}\right) \in \prod_{j \in J} \mathcal{A}_{j}$. This follows immediately from the observation that two elements $\left(a_{j}\right),\left(b_{j}\right) \in \prod_{j \in J} \mathcal{A}_{j}$ are adjacent in $\Gamma_{\left(x_{j}\right)}\left(\prod_{j \in J} \mathcal{A}_{j}\right)$ if and only if $\left(a_{j}\right)\left(b_{j}\right)=\left(x_{j}\right)$ in the groupoid $\prod_{j \in J} \mathcal{A}_{j}$, if and only if $a_{j} b_{j}=x_{j}$ in $\mathcal{A}_{j}$ for every $j \in J$, if and only if $a_{j} \in N_{\Gamma_{x_{j}}\left(\mathcal{A}_{j}\right)}\left(b_{j}\right)$ for every $j \in J$, if and only if the elements $\left(a_{j}\right),\left(b_{j}\right) \in \prod_{j \in J} \mathcal{A}_{j}$ are adjacent in $\prod_{j \in J} \Gamma_{x_{j}}\left(\mathcal{A}_{j}\right)$.

The following corollary emphasizes (from the standpoint of category theory) ways in which the above results confirm the given definitions of "quotient-C.S.P." and "direct product" of C.S.P.s. Recall that, if $\sim$ is a congruence on a commutative groupoid $\mathcal{A}$, then the function mapping an element $a \in \mathcal{A}$ to the element $\bar{a} \in \mathcal{A} / \sim$ is a groupoid-homomorphism.

Corollary 3.10. The following statements hold.

(1) Suppose that $\sim$ is a congruence on a C.S.P. $\mathscr{G}$, and let $\psi: \mathscr{G} \rightarrow \mathscr{G} / \sim$ be the C.S.P.-homomorphism that maps every element to its equivalence class. Then $\mathscr{G} / \sim$ satisfies the following universal property: If $\mathscr{G}^{\prime}$ is a C.S.P. and $\sigma: \mathscr{G} \rightarrow \mathscr{G}^{\prime}$ is a C.S.P.-homomorphism such that $\sigma(a)=\sigma(b)$ whenever $a \sim b$ then there exists a unique C.S.P.-homomorphism $\mu: \mathscr{G} / \sim \rightarrow \mathscr{G}^{\prime}$ such that $\mu \psi=\sigma$. Moreover, $\mathscr{G} / \sim \cong \sigma(\mathscr{G})$.

(2) If $\left\{\mathscr{G}_{j}\right\}_{j \in J}$ is a family of C.S.P.s then there exists a family of C.S.P.homomorphisms $\left\{\rho_{i}: \prod_{j \in J} \mathscr{G}_{j} \rightarrow \mathscr{G}_{i} \mid i \in J\right\}$ that satisfies the following universal property: If $\mathscr{G}$ is a C.S.P. and $\left\{\sigma_{i}: \mathscr{G} \rightarrow \mathscr{G}_{i} \mid i \in J\right\}$ is a family of C.S.P.-homomorphisms then there exists a unique C.S.P.-homomorphism $\sigma: \mathscr{G} \rightarrow \prod_{j \in J} \mathscr{G}_{j}$ such that $\rho_{i} \sigma=\sigma_{i}$ for every $i \in J$.

Proof. Note that $\psi$ is a C.S.P.-homomorphism by Theorem 3.7 since, by the above comments together with Theorem 3.4, it is the image under $\mathcal{D}$ of a homomorphism of the corresponding commutative groupoids. The universal property given in (1) holds similarly (since the corresponding statement holds for commutative groupoids). Also, the "moreover" statement can be verified using Proposition 3.5 since the corresponding statement holds for commutative groupoids.

For (2), the corresponding universal property holds for direct products of commutative groupoids (with $\rho_{i}$ being the usual projection mapping), so the result follows 
by Theorem 3.9 together with the observation that the functor $\mathcal{D}$ is bijective on objects and morphisms.

Acknowledgment. The author would like to thank the referee for carefully reading the paper and for pointing out an error that was subsequently corrected.

\section{References}

[1] D. F. Anderson, M. C. Axtell and J. A. Stickles, Jr., Zero-divisor graphs in commutative rings, in Commutative Algebra, Noetherian and Non-Noetherian Perspectives (M. Fontana, S.-E. Kabbaj, B. Olberding, I. Swanson, Eds.), Springer-Verlag, New York, (2011), 23-45.

[2] D. F. Anderson and P. S. Livingston, The zero-divisor graph of a commutative ring, J. Algebra, 217(2) (1999), 434-447.

[3] D. F. Anderson and S. B. Mulay, On the girth and diameter of a zero-divisor graph, J. Pure Appl. Algebra, 210(2) (2007), 543-550.

[4] M. Axtell, J. Coykendall and J. Stickles, Zero-divisor graphs of polynomials and power series over commutative rings, Comm. Algebra, 33(6) (2005), 20432050 .

[5] I. Beck, Coloring of commutative rings, J. Algebra, 116(1) (1988), 208-226.

[6] R. H. Bruck, A Survey of Binary Systems, Springer-Verlag, Berlin, 1958.

[7] J. H. Conway, A simple construction for the Fischer-Griess monster group, Invent. Math., 79(3) (1985), 513-540.

[8] J. Coykendall, S. Sather-Wagstaff, L. Sheppardson and S. Spiroff, On zero divisor graphs, in Progress in Commutative Algebra II: Closures, Finiteness and Factorization (C. Francisco et al., Eds.), Walter de Gruyter, Berlin, (2012), 241-299.

[9] F. R. DeMeyer, T. McKenzie and K. Schneider, The zero-divisor graph of a commutative semigroup, Semigroup Forum, 65(2) (2002), 206-214.

[10] R. Halaš and M. Jukl, On Beck's coloring of posets, Discrete Math., 309(13) (2009), 4584-4589.

[11] B. A. Hausmann and O. Ore, Theory of quasi-groups, Amer. J. Math., 59(4) (1937), 983-1004.

[12] T. W. Hungerford, Algebra, Springer-Verlag, New York, 1974.

[13] J. Ježek and T. Kepka, A note on medial division groupoids, Proc. Amer. Math. Soc., 119(2) (1993), 423-426.

[14] V. Joshi and A. Khiste, On the zero divisor graphs of pm-lattices, Discrete Math., 312 (2012), 2076-2082. 
[15] A. D. Keedwell, Uniform P-circuit designs, quasigroups, and Room squares, Utilitas Math., 14 (1978), 141-159.

[16] U. Knauer, Algebraic Graph Theory: Morphisms, Monoids and Matrices, De Gruyter Studies in Mathematics, 41, Walter de Gruyter \& Co., Berlin, 2011.

[17] A. Kotzig, Groupoids and partitions of complete graphs, in Combinatorial Structures and their Applications (Proc. Colloq. Calgary 1969), Gordon and Breach, New York, (1970), 215-221.

[18] D. $\mathrm{Lu}$ and $\mathrm{T}$. Wu, The zero-divisor graphs of posets and an application to semigroups, Graphs Combin., 26(6) (2010), 793-804.

[19] T. G. Lucas, The diameter of a zero divisor graph, J. Algebra, 301(1) (2006), 174-193.

[20] B. V. Novikov, On decomposition of commutative Moufang groupoids, Quasigroups Related Systems, 16(1) (2008), 97-101.

[21] S. P. Redmond, The zero-divisor graph of a noncommutative ring, Int. J. Commut. Rings, 1(4) (2002), 203-211.

[22] I. M. Wanless and E. C. Ihrig, Symmetries that latin squares inherit from 1-factorizations, J. Combin. Des., 13(3) (2005), 157-172.

\section{John D. LaGrange}

Division of Natural Science and Mathematics

Lindsey Wilson College

Columbia, KY 42728-1223, USA

e-mail: lagrangej@lindsey.edu 